\title{
Antimicrobial resistance development following surgical site infections
}

\author{
DANIELA CĂLINA $^{1^{*}}$, ANCA OANA DOCEA ${ }^{2 *}$, LUCICA ROSU $^{3 *}$, \\ OVIDIU ZLATIAN ${ }^{3 *}$, ALEXANDRA FLORIANA ROSU $^{4 *}$, FLORIN ANGHELINA $^{5^{*}}$, \\ OTILIA ROGOVEANU ${ }^{6 *}$, ANDREEA LETIȚIA ARSENE ${ }^{7 *}$, ALINA CRENGUȚA NICOLAE $^{8^{*}}$, \\ CRISTINA MANUELA DRĂGOI ${ }^{8 *}$, JOHN TSIAOUSSIS $^{9 *}$, ARISTIDES M. TSATSAKIS ${ }^{10^{*}}$, \\ DEMETRIOS A. SPANDIDOS ${ }^{11^{*}}$, NIKOLAOS DRAKOULIS ${ }^{12^{*}}$ and ELIZA GOFITA ${ }^{2 *}$
}

\begin{abstract}
Departments of ${ }^{1}$ Clinical Pharmacy, ${ }^{2}$ Toxicology, ${ }^{3}$ Microbiology, ${ }^{4}$ Gastroenterology, ${ }^{5}$ Otorhinolaryngology and ${ }^{6}$ Physiotherapy, University of Medicine and Pharmacy of Craiova, 200349 Craiova; Departments of ${ }^{7}$ Pharmaceutical Microbiology and ${ }^{8}$ Biochemistry, Carol Davila University of Medicine and Pharmacy, 020021 Bucharest, Romania; ${ }^{2}$ Laboratory of Anatomy, Medical School of Heraklion, University of Crete; ${ }^{10}$ Laboratory of Toxicology, and ${ }^{11}$ Laboratory of Clinical Virology, Medical School, University of Crete, Heraklion 71003; ${ }^{12}$ Research Group of Clinical Pharmacology and Pharmacogenomics, Faculty of Pharmacy, School of Health Sciences, National and Kapodistrian University of Athens, Athens 15771, Greece
\end{abstract}

Received September 13, 2016; Accepted December 2, 2016

DOI: $10.3892 / \mathrm{mmr} .2016 .6034$

\begin{abstract}
Surgical site infections (SSIs) determine an increase in hospitalization time and antibiotic therapy costs. The aim of this study was to identify the germs involved in SSIs in patients from the Clinical Emergency County Hospital of Craiova (SCJUC) and to assess their resistance to antimicrobials, with comparisons between surgical wards and the intensive care unit (ICU). The biological samples were subjected to classical bacteriological diagnostics. Antibiotic resistance was tested by disc diffusion. We used hierarchical clustering as a method to group the isolates based upon the antibiotic resistance profile. The most prevalent bacterial species isolated were Staphylococcus aureus (S. aureus; 50.72\%), followed by Escherichia coli (E. coli; 17.22\%) and Pseudomonas aeruginosa $; 10.05 \%)$. In addition, at lower percentages, we isolated glucose-non-fermenting, Gram-negative bacteria and other Enterobacteriaceae. The antibiotic resistance varied greatly between species; the most resistant were the non-fermenting Gram-negative rods. E. coli exhibited lower resistance to third
\end{abstract}

Correspondence to: $\operatorname{Dr}$ Anca Oana Docea, Department of Toxicology, University of Medicine and Pharmacy of Craiova, 2 Petru Rareş Street, 200349 Craiova, Romania

E-mail: ancadocea@gmail.com

*Contributed equally

Abbreviations: SSIs, surgical site infections; ICU, intensive care unit

Key words: surgical site infections, antibiotic resistance, microbiology, healthcare associated infections generation cephalosporins, quinolones and carbapenems. By contrast, Klebsiella was resistant to many cephalosporins and penicillins, and to a certain extent to carbapenems due to carbapenemase production. The non-fermenting bacteria were highly resistant to antibiotics, but were generally sensitive to colistin. S. aureus was resistant to ceftriaxone (100\%), penicillin $(91.36 \%)$, amoxicillin/clavulanate $(87.50 \%)$, amikacin $(80.00 \%)$ and was sensitive to levofloxacin, doxycycline, gentamycin, tigecycline and teicoplanin. The Enterobacteriaceae resistance was only slightly higher in the ICU, particularly to carbapenems (imipenem, $31.20 \%$ in the ICU vs. $14.30 \%$ in the surgical wards; risk ratio $=2.182$ ). As regards Staphylococcus species, but for non-fermenting bacteria, even if the median was almost the same, the antibiotic resistance index values were confined to the upper limit in the ICU. The data gathered from this study may help infection control teams to establish effective guidelines for antibiotic therapies in various surgical procedures, in order to minimize the risk of developing SSIs by the efficient application of the anti-infection armamentarium.

\section{Introduction}

Abdominal surgical wound infections in adults are defined as infections that occur within the maximum time of 30 days postsurgery and contribute to increased post-surgical morbidity and mortality. The most common complications are abscesses, wound infections and necroses (1). Surgical site infections (SSIs) represent $15 \%$ of all nosocomial infections (2) and are associated with prolonged hospitalization time and increased antibiotic therapy costs (3). The most frequently involved microorganisms include: Staphylococcus aureus (S. aureus; 15-20\%), Gram-negative bacilli, coagulase-negative staphylococci, Enterococcus spp. and Escherichia coli (E. coli) (4). From these, methicillin-resistant $S$. aureus (MRSA) represents 
$50 \%$ of hospital-acquired infections in the United States and Europe, and these infections very difficult to treat due to their resistance to multiple antibiotics (5).

The Centers for Disease Control and Prevention (CDC) (6) classify SSIs into three major categories: i) superficial infections, which are localized to the skin and subcutaneous tissue, and are characterized locally by redness, pain, warmth and swelling, and are resolved by local incision and the discharge of the pus; ii) deep incisional infections, affecting muscles and fascia with the presence of abscess, which require the surgical excision of deep wound edges; and iii) the infection of abdominal organs or anatomical spaces, which require surgical procedures in locations other than the initial incision site (6).

The objectives of this study were the following: i) the identification of germs that produced surgical wound infections in patients from the Clinical Emergency County Hospital of Craiova (Craiova Romania) and to assess their resistance to antimicrobials; and ii) perform a comparison of antibiotic resistance profiles between surgical wards and the intensive care unit (ICU), in order to determine the most effective therapeutic protocols.

\section{Materials and methods}

Patient data. This study was conducted between September 2015 and September 2016, and included a total of 165 patients (male:female ratio, 2.17) aged between 18 and 87 years, hospitalized at the General Surgery departments of the Clinical Emergency County Hospital of Craiova, and diagnosed clinically with SSIs following various surgical interventions. We collected discharge, liquid drainage from superficial and deep surgical wounds and pus. Each patient included in the study provided written informed consent. The study was conducted in accordance with the World Medical Association Declaration of Helsinki and was approved by the Institutional Ethics Committee of Clinical Emergency Hospital of Craiova.

Microbiological evaluation. The strains were identified by classical bacteriological diagnosis and the antimicrobial testing was performed by disc diffusion according to the guidelines of the Clinical and Laboratory Standards Institute (CLSI) (7).

Statistical analysis. The results of antimicrobial testing were stored and analyzed using Whonet 5.6 software (World Health Organisation, Geneva, Switzerland). Statistical analyses were performed using the Statistical Package for the Social Sciences (SPSS, Inc., Chicago, IL, USA) 20.0 software (IBM Corp., Armonk, NY, USA). Antibiotic resistance was expressed as the ratio between the number of isolates tested as resistant over the number of isolates tested to the antibiotic.

For each isolate, we calculated the multiple antibiotic resistance (MAR) index as the ratio between the number of antibiotics at which the isolate was resistant over the number of antibiotics tested for that isolate. Comparative analyses of continuous variables were made using the Student's t-test for two groups and one-way analysis of variance (ANOVA) for more than two groups. A value of $\mathrm{P}<0.05$ was considered to indicate a statistically significant difference.
Hierarchical clustering was used as a method to group the isolates based upon the antibiotic resistance profile, knowing that strains spreading from a patient to another suffer mutations in the mechanisms of antibiotic resistance depending on the antibiotic treatment received by the patient. Thus, the degree of relatedness of the antibiotic profile is an indication of the degree of the genetic relatedness of the strains. Hierarchical clustering was demonstrated to be a reliable method for constructing filiation trees of genetic relatedness (8).

We performed hierarchical clustering as previously described by $\mathrm{Xu}$ et al (9). We measured the diameters of inhibition areas around each antibiotic disk on the Petri dish and entered them into the hierarchical clustering procedure of SPSS. For clustering, we used Ward's minimum variance method. Each isolate was assigned to a specific cluster based on their zone diameters.

\section{Results}

Samples were collected from 24 patients with superficial SSIs, 42 patients with deep incisional infections and 99 patients with deep abdominal infections.

A total of 209 bacterial strains were isolated from the patients included in this study $(n=165)$. The most prevalent bacterial species isolated were $S$. aureus $(50.72 \%)$, followed by E. coli (17.22\%) and Pseudomonas aeruginosa (10.05\%). Proteus mirabilis, non-fermenting Gram-negative rods, Klebsiella pneumoniae, Enterobacter aerogenes and Proteus vulgaris were also isolated, although at lower percentages (Fig. 1).

The comparison of the antibiotic resistance index by species (Fig. 2A), revealed that the differences between species were statistically significant (ANOVA, $\mathrm{P}<0.001$ ), as the non-fermenting bacteria were the most resistant (median MAR, 0.91) and E. coli was the least resistant (median MAR, 0.40). Pseudomonas was included in the non-fermenting bacteria category due to the low number of strains.

A comparison between the resistance of various bacterial species in the ICU and surgical wards was performed (Fig. 2B). For Enterobacteriaceae, the resistance was only slightly higher in the ICU. As regards Staphylococcus species, but for nonfermenting bacteria, even if the median MAR was almost the same, the antibiotic resistance index values were confined to the upper limit in the ICU. For all species, the p-values from the Student's t-test performed on the MAR index between surgical wards and the ICU were $<0.01$.

The $S$. aureus strains were highly resistant to ceftriaxone $(100 \%)$, penicillin $(91.36 \%)$, amoxicillin/clavulanate $(87.50 \%)$, amikacin $(80.00 \%)$ and amoxicillin $(83.33 \%)$. The resistance to cefoxitin was $40 \%$. Low resistance rates were encountered to levofloxacin (37.10\%), doxycycline $(31.20 \%)$, gentamycin $(17.01 \%)$, tigecycline $(6.90 \%)$ and teicoplanin (6.90\%) (Fig. 3A).

After performing the antimicrobial susceptibility testing of the isolated E. coli strains (Fig. 3B), an elevated resistance to cefuroxime $(89.50 \%)$, cefepime $(84.20 \%)$ and cefazoline $(77.80 \%)$ was registered. A moderate resistance was observed to amoxicillin/clavulanate $(42.10 \%)$, ceftriaxone $(42.10 \%)$, aztreonam $(31.60 \%)$ and ciprofloxacin $(31.60 \%)$. The strains of $E$. coli exhibited a low resistance 


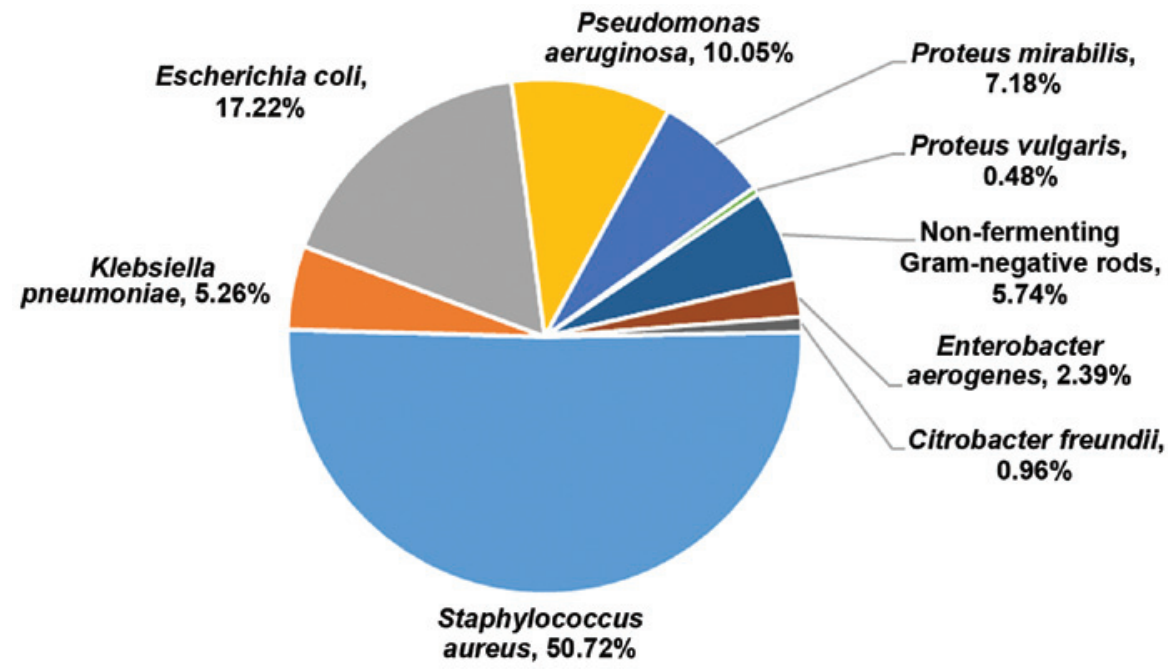

Figure 1. Prevalence of bacterial species involved in surgical site infections.
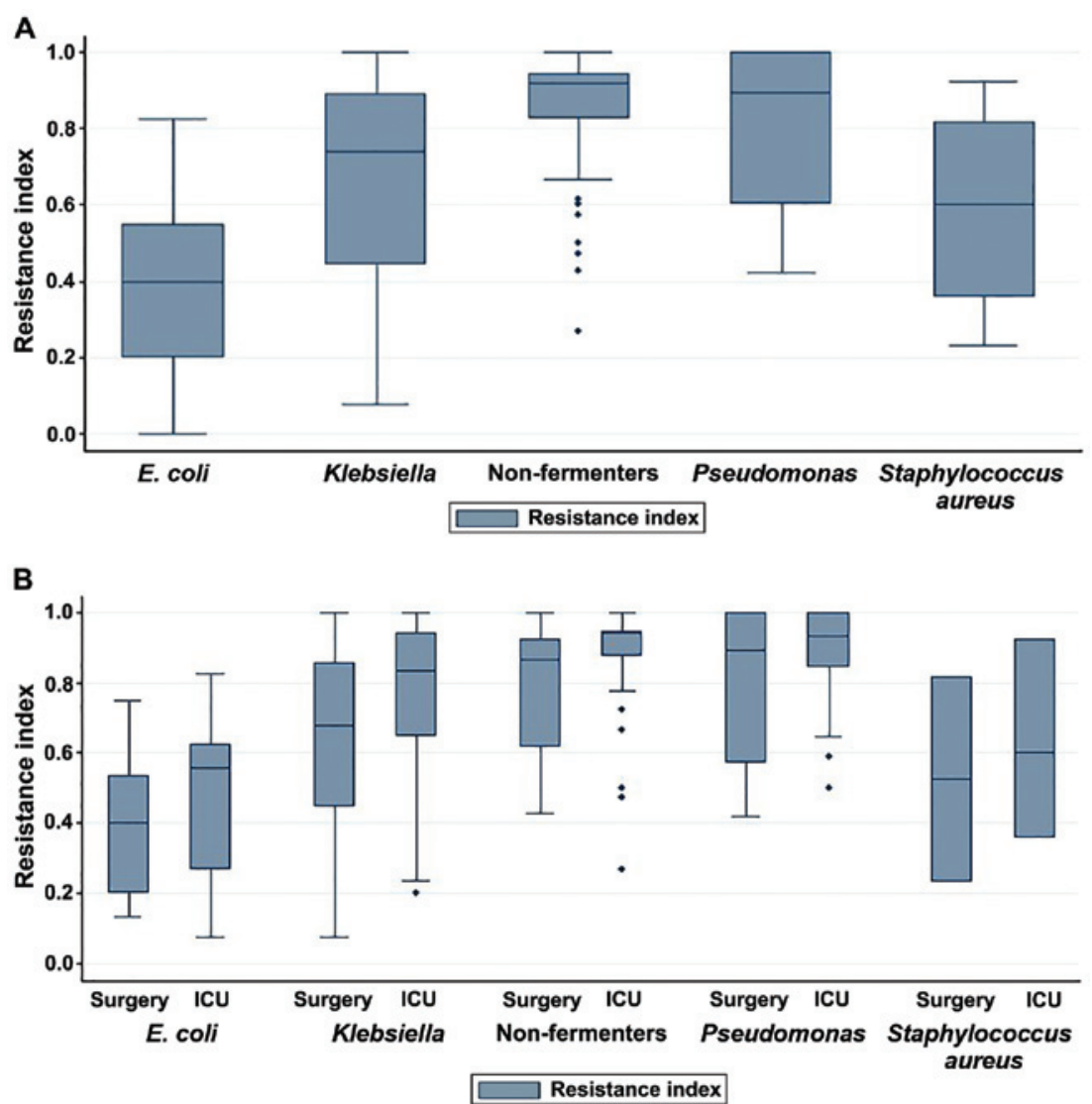

Figure 2. (A) The antibiotic resistance index of the isolated species; (B) comparison of the antibiotic resistance index of the isolated species isolated from ICU and surgical wards. ICU, intensive care unit.

to trimethoprim/sulfamethoxazole (19.00\%) and zero resistance to amikacin and ertapenem.

The isolated Klebsiella strains were found to be 100\% resistant (Fig. 3C) to ampicillin, cefuroxime and ticarcillin/ clavulanate, and resistant to cefazolin (90.50\%), amoxicillin/ clavulanate $(87,50 \%)$, cefpirome $(83.30 \%)$, aztreonam $(81.80 \%)$, cefepime $(78.30 \%)$, piperacillin with tazobactam $(62.50 \%)$, ciprofloxacin $(52.40 \%)$, amikacin $(50,00 \%)$ and sulfametoxazole withtrimethoprim(45.50\%).Alowlevelofresistancewasobserved to cefoperasone-sulbactam $(16.70 \%)$, imipenem $(25.00 \%)$, tigecycline $(25.00 \%)$ and tobramycin $(25.00 \%)$.

The glucose non-fermenting bacteria were highly resistant to antibiotics (Fig. 3D). For example, the resistance was very high to fosfomycin (98.35\%), cefepime $(94.40 \%)$, meropenem (90.00\%), amoxicillin/clavulanate (83.30\%), aztreonam $(78.30 \%)$ and ertapenem $(76.50 \%)$. There was profound resistance to piperacillin-tazobactam $(69.60 \%)$, ciprofloxacin $(62.50 \%)$, imipenem $(59.10 \%)$ and gentamycin (57.10\%), but 
A

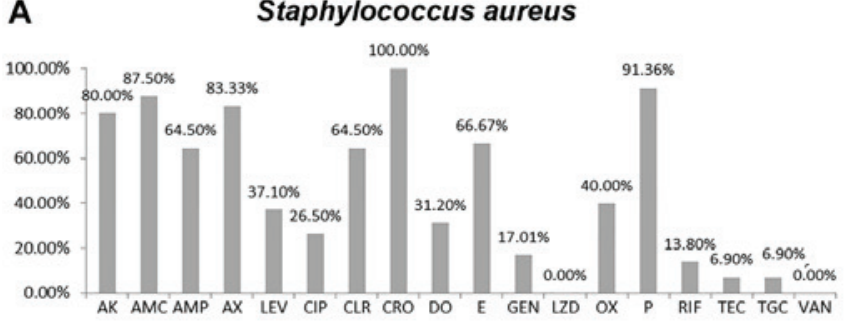

\section{B}

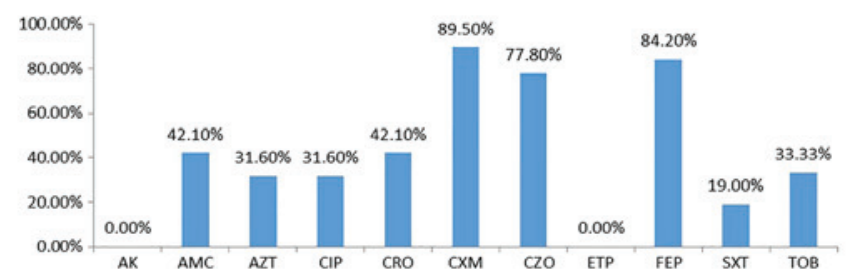

C

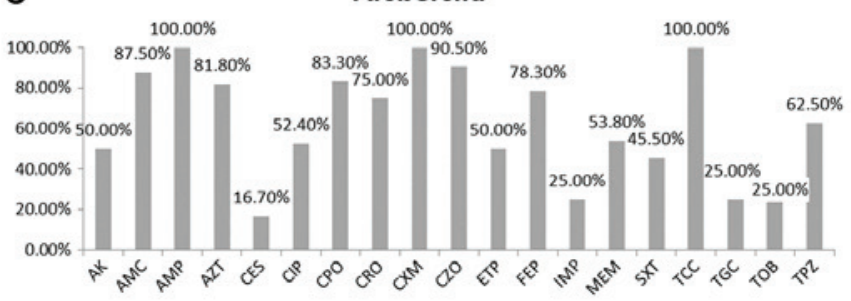

D

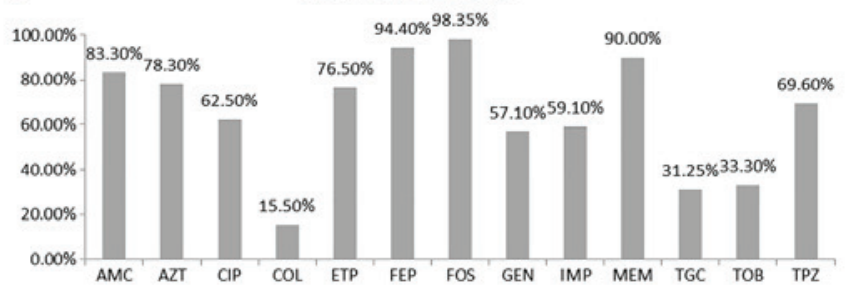

Figure 3. Antibiotics resistance profile of (A) Staphylococcus aureus, (B) Escherichia coli, (C) Klebsiella pneumoniae and (D) non-fermenting Gram-negative rods (non-fermenters). AK, amikacin; AMC, amoxicillin/clavulanate; AMP, ampicillin; AZT, aztreonam; AX, amoxicillin; CES, cefoperasone sulbactam; CIP, ciprofloxacin; CLR, clarithromycin; COL, colistin; CPO, cefpirome; CRO, ceftriaxone; CXM, cefuroxime; CZO, cefazoline; DO, doxycycline; E, erythromycin; ETP, ertapenem; FEP, cefepim; FOS, fosfomycin; GEN, gentamycin; IMP, imipenem; LEV, levofloxacin, LZD, linezolid; MEM, meropenem; OX, oxacillin; P, penicillin; RIF, rifampin; SXT, trimethoprim/sulfamethoxazole; TEC, teicoplanin; TCC, ticarcillin/clavulanate; TGC, tigecycline; TOB, tobramycin; TPZ, piperacillin-tazobactam; VAN, vancomycin.
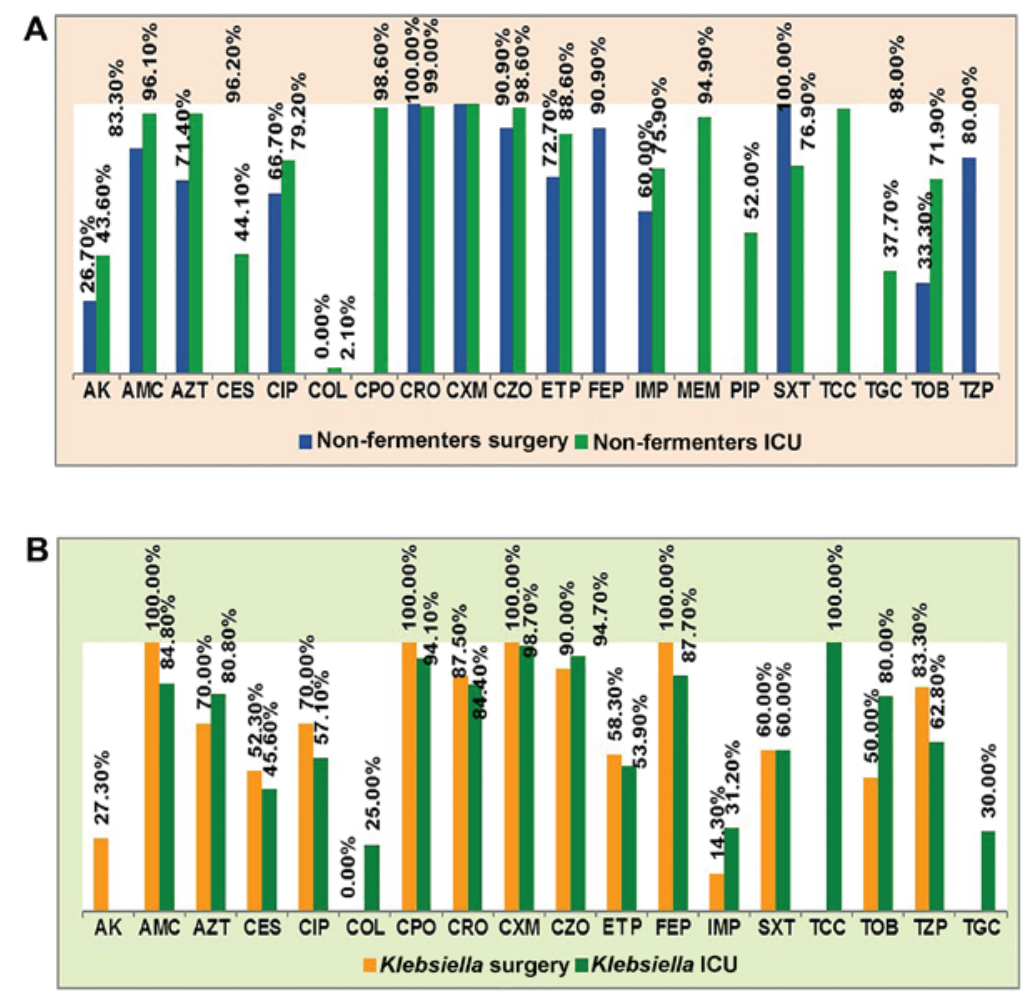

Figure 4. Antibiotics resistance profile of (A) non-fermenting Gram negative rods (non-fermenters) and (B) Klebsiella pneumoniae in surgery and ICU wards. AK, amikacin; AMC, amoxicillin/clavulanate; AZT, aztreonam; CES, cefoperasone sulbactam; CIP, ciprofloxacin; COL, colistin; CPO, cefpirome; CRO, ceftriaxone; CXM, cefuroxime; CZO, cefazoline; ETP, ertapenem; FEP, cefepim; IMP, imipenem; MEM, meropenem; PIP, piperacillin; SXT, trimethoprim/sulfamethoxazole; TCC, ticarcillin/clavulanate; TGC, tigecycline; TOB, tobramycin; TPZ, piperacillin-tazobactam.

low resistance rates were encountered for tobramycin (33.30\%), tigecycline $(31.25 \%)$ and colistin $(15.50 \%)$. The non-fermenting bacteria from patients in the ICU were generally more resistant than those from patients in surgical wards (Fig. 4A).

The resistance of Klebsiella strains differed between the surgical and ICU wards for imipenem $[31.20 \%$ in ICU vs. $14.30 \%$ in surgical wards, risk ratio $(R R)=2.182$, piperacillin with tazobactam (62.80 vs. $83.30 \%, R R=0.754)$, tobramycin ( 80.00 vs. $50.00 \%, \mathrm{RR}=1.600)$ and amikacin (0 vs. $27.00 \%)$ and colistin $(25.00$ vs. $0 \%)$. There were no significant differences as regards resistance to cefuroxime, cefpirome and amoxycillin/clavulanate. We can see that for 


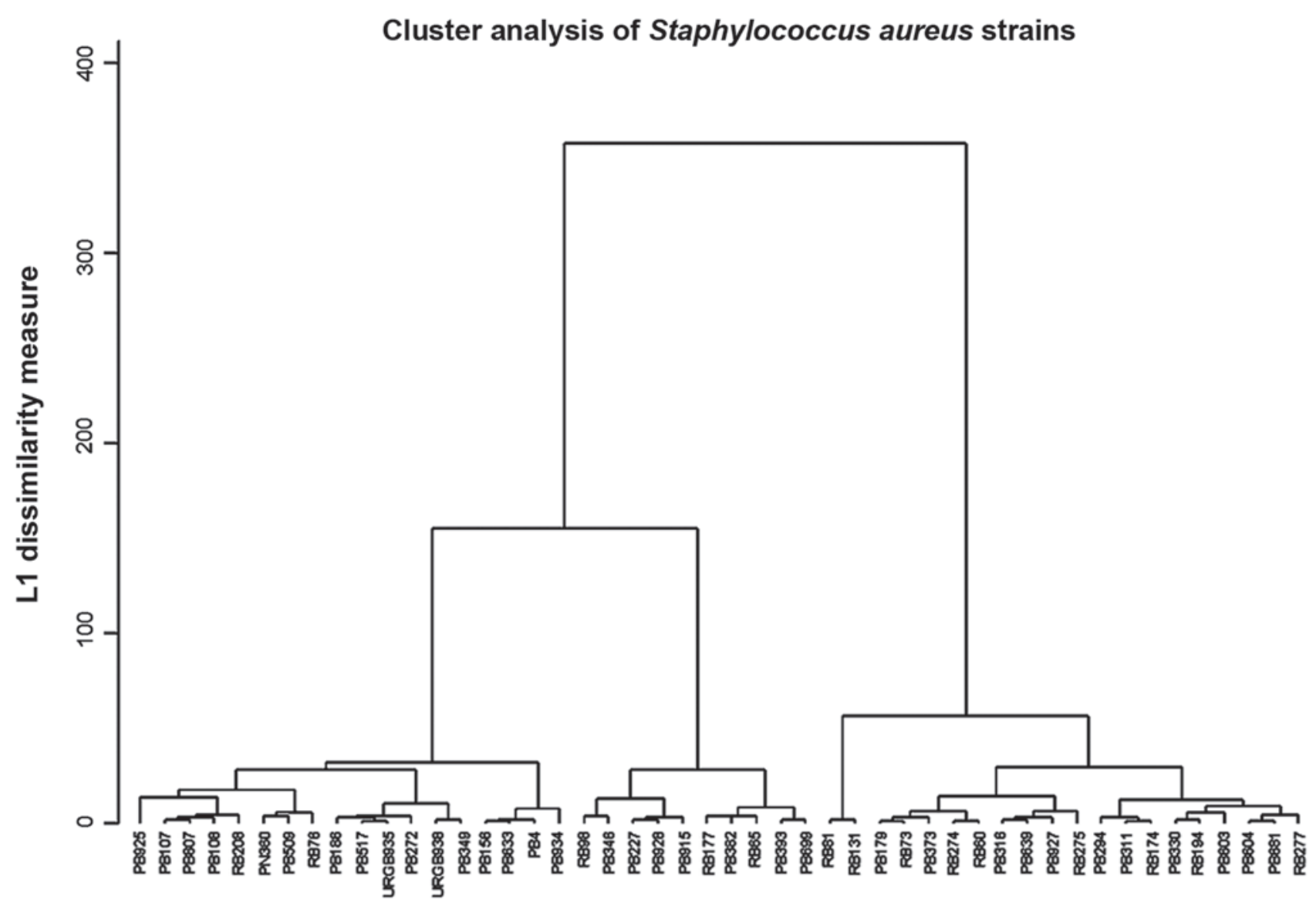

Figure 5. Hierarchical cluster analysis of resistance of Staphylococcus aureus strains (Ward's minimum variance method).

almost all antibiotics $>50 \%$ of the ICU strains were resistant (Fig. 4B).

The clustering analysis revealed 12 clusters for S. aureus; 6 in the sensitive area, probably low-resistant $S$. aureus from the aeromicroflora and 6 in the high resistance area, the MRSA (Fig. 5).

The E. coli and Klebsiella strains had a heterogenous resistance, distributed in 11 clusters over the whole interval, as Klebsiella can easily acquire resistance plasmids from other enterobacteria (Fig. 6).

\section{Discussion}

The knowledge of the antibiotic resistance of bacteria recovered from SSIs is critical in for the optimization of the prophylactic antibiotic therapy of surgical maneuvers, in an effort to avoid the selection of multi-resistant microorganisms. Although the emergence of resistant strains is a natural phenomenon which cannot be avoided, the transformation of resistant strains into resistant populations is favored by non-rational antibiotic therapy. It is well known that the ICU departments are the centers of dissemination of multi-resistant strains in hospitals, a fact that underlines the importance to compare the differences in resistance between ICU and surgical wards.

In the case of Klebsiella, known as a bacterium that collects easily resistance plasmids, the additional resistance observed in surgical wards may possibly reflect the prophylactic usage of antibiotics routinely administered prior to surgery, that may also induce resistance to other bacteria, that would survive in the hospital environment and would lastly transmit the resistance genes to Klebsiella strains (10). The $52.40 \%$ resistance to ciprofloxacin is important in treating the pleural infections, as ciprofloxacin penetrates well in the pleural space (11).

Although increased bacterial resistance would be expected in ICUs due to the higher usage of antibiotics, the differences in the antibiotic resistance of bacterial strains derived from ICU or surgical wards was minor in this study. The differences in resistance were minor for cephalosporins. The highest difference was observed for cefepime (100\% in ICU vs. $87.50 \%$ in surgical wards). These data confirm those of an earlier study that found small differences in resistance between ICU and surgical wards to 1st and 2nd generation cephalosporins, and higher for 4th generation cephalosporins (84.2 vs. $69.82 \%$ for cefepime) (4). This may suggest that the highly resistant Klebsiella strains from the ICU have already colonized the surgical wards, due to the fact that many surgical patients are transferred from the surgery room directly to the ICU, where they spend a few days until they are transported back into the surgical ward. Furthermore, the differences identified in carbapenem resistance ( 31.20 vs. $14.30 \%$ for imipenem) were probably due to the high prescription rate in the ICU (12). It has been demonstrated that if $\beta$-lactams are used for a prolonged period of time, the resistance rapidly increases within several years $(13,14)$.

In our study, non-fermenting bacteria had generally higher resistance rates than other bacteria, particularly in 4th generation 


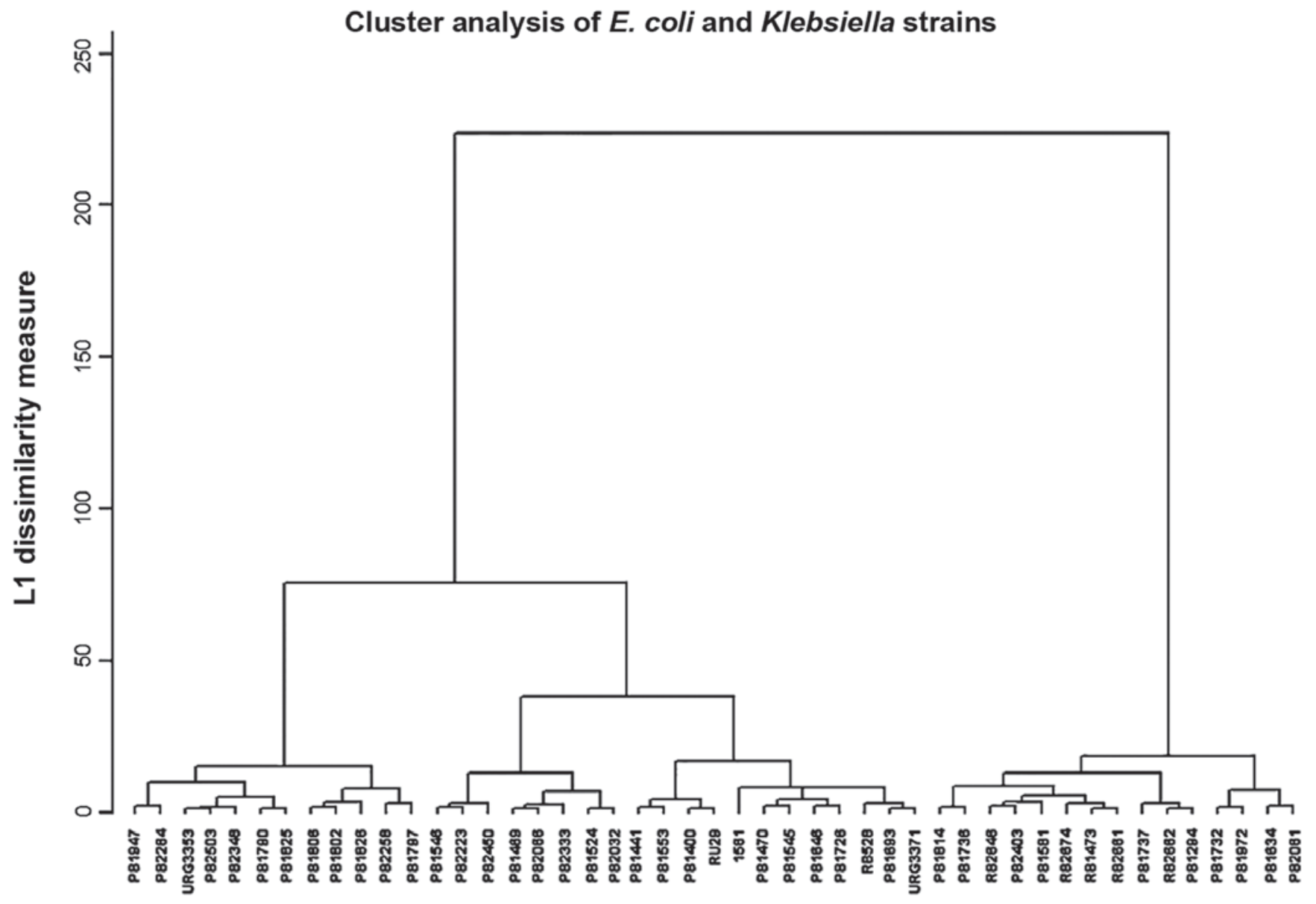

Figure 6. Hierarchical cluster analysis of resistance of Escherichia coli and Klebsiella pneumoniae strains (Ward's minimum variance method).

cephalosporins, penicillins with inhibitors and carbapenems. A notable difference between resistance to meropenem $(90.00 \%)$ and imipenem $(59.10 \%)$ was detected, that can be correlated with a greater prescription of meropenem. The aminoglicosides resistance was also high (57.10\% to gentamycin), but below that for cephalosporins. The prophylactic use of gentamycin may be therefore justified (15). Nevertheless, the cephalosporins are still recommended as prophylactic antibiotherapy in abdominal surgery (16). Minocycline, an old antibiotic with proven safety, that has been reported in the last few years to express improved efficacy in preventing infections with Acinetobacter baumannii would represent a further effective alternative (17).

The resistance differences between ICU and surgical wards were greater for non-fermenting bacteria than for Klebsiella. This can be at least partly explained by the fact that in ICU, many patients may have extremely low immunity due to their medical conditions (18). They may therefore have an elevated risk of acquiring common environmental pathogen infections, which would not infect patients with normal immunity (e.g., from surgical wards) and possess high natural resistance to antibiotics, such as Acinetobacter species (19) or opportunistic fungal agents, scuh as Fusarium (20). The fact that Pseudomonas is spread in various wards of the hospital and Acinetobacter affects mainly patients from ICU can explain the greater prevalence of Pseudomonas (10.05\%) compared with other non-fermenting bacteria (5.74\%). This difference is constantly reported by studies on all infections, not only in
SSIs (21). The high usage of antibiotics in the ICU promotes the higher resistance of bacteria isolated from these patients.

The isolation of MRSA from surgical wounds has been shown to be related to lower chances of primary healing and delayed healing (22). The current method for testing MRSA is to test the resistance to cefoxitin (23), which is also a surrogate test for resistance to oxacillin. Hence, we can conclude that $40 \%$ of the $S$. aureus isolates were MRSA. MRSA can establish within hospitals and becomes difficult to control. Its survival is promoted probably by the high usage of antibiotics, that can induce the transformation of methicillin susceptible $S$. aureus (MSSA) in MRSA (24). In our study, the prevalence of MRSA was $20.29 \%$ $[$ MRSA prevalence $=$ MRSA rate $(40 \%) \times$ S . aureus prevalence $(50.72 \%)$ ]. This is similar with other observations reporting MRSA prevalence of $28.50 \%$ in SSIs (25).

A recent finding is that many apparently susceptible $S$. aureus strains actually contain small populations of mecApositive cells (26). Many patients are colonized at admission with MSSA, located mostly in the pharynx, but also in other sites (3). For example, some of the patients in the present study had acne, from which $S$. aureus was isolated (27). These MRSA subpopulations can be selected through exposure to cefoxitin or oxacillin up to $48 \mathrm{~h}$ (28). Probably, the real prevalence of MRSA in SSI is $>20.29 \%$ (29).

A small difference between resistance to amoxicillin alone $(83.33 \%)$ and resistance to amoxicillin/clavulanate combination $(87.50 \%)$ was documented, indicating that only a 
few strains of Staphylococcus may secrete $\beta$-lactamases in the study samples. The resistance to quinolones has been reported to emerge following treatment with ciprofloxacin (30). In the current study, a ciprofloxacin resistance of $26.50 \%$ was found, possibly as many surgeons use quinolones as prophylactic therapy. The low resistance to rifampin of $13.80 \%$ can be explained as this is a reserve antibiotic, for cases with tuberculosis. This is in accordance with other reports; for example, Peterson et al reported a $15.50 \%$ resistance of $S$. aureus strains to rifampin (31).

Profiling the resistance to antimicrobials using the diameters of inhibition areas obtained by disk diffusion is widely used in studies of epidemiology and strain homology (32). The usual analysis methods of antibiotic resistance do not take advantage of the continuous nature of the inhibition zone diameter, as these methods analyze the binary variable of resistance. Cluster analysis can use continuous variables on large scale and provides homology trees that can help us understand the relatedness of the isolates (33).

The acquisition of resistance genes by Klebsiella strains, a very frequent observation in a hospital environment, can be demonstrated by cluster analysis. In Fig. 6 we can observe two big clusters, the strains from the 2 nd cluster had a higher resistance index. Possibly, these represent hospital strains and the next level of clusters contains strains that acquired genes for extended-spectrum $\beta$-lactamases (ESBLs) and carbapenemases. Strains carrying carbapenemase genes are in the third cluster (Fig. 6). The next level of clustering, shows three 3rd degree clusters that may correspond to different resistance mechanisms. It is known that the ESBL production confer resistance to many penicillin derivatives and cephalosporins as the carbapenemases production render the bacteria resistant to most $\beta$-lactams. Other mechanisms such as porin loss (impermeability) can also lead to very high antibiotic resistance. The clusters of 1st, 2nd and 3rd degree may reflect the distribution of antibiotic resistance mechanisms in the Enterobacteriaceae population in the current study hospital rather than relatedness due to transmission from patient to patient. Further levels of the clustering analysis can be used for this purpose, and can be observed similarity of resistance profiles between patients from ICU and surgical wards could be due to ICU admission of patients shortly after the surgery, where they may stay for several days, during which they may acquire highly resistant strains.

Analysis of the Staphylococcus resistance cluster dendrogram, reveals that the MRSA clearly differentiate from the sensitive staphylococci. There is a hierarchy in resistance of MRSA which suggests acquisition of supplementary resistance genes (Fig. 5). These could be erm genes that produce of the ribosomal methylation.

In conclusion, up to $40 \%$ of surgeries are associated to postoperative infectious complications.

The most common bacterial pathogen of SSIs is S. aureus, followed by gram-negative organisms. This high incidence can be partially be explained by the high load of $S$. aureus in air flora, the colonization of the patients at admission and probably to other unidentified perioperative factors.

The bacteria isolated from ICU wards have a higher resistance to certain antimicrobials, especially carbapenems, compared with those from surgical wards. The use of these agents should be avoided in ICU wards to prevent the development of the resistance.

Beyond the detection of antibiotic resistance, the molecular mechanisms that underlie resistance have been elucidated by specific phenotypic and molecular methods, such as PCR and mass-spectroscopy.

The drug sensitivity profile expressed as diameters of inhibition areas of antibiotics in the diffusimetric method can be used for phenotypic typing and epidemiological tracing of hospital strains. This can contribute to antibiotic stewardship measures, in hospitals that cannot afford expensive analyzers that can type the strains as mass spectrometers or DNA sequencers.

The anti-infectious therapy optimized based upon antibiotic resistance of the bacterial strains will improve the quality of medical care, by discarding the antibiotics which loosed their efficacy.

The data gathered from this study can help the infection control team to establish effective guidelines for antibiotic therapies in various surgical procedures, with the aim to minimize the risk of developing SSIs and to the correct and efficient use of the anti-infectious armamentarium.

\section{References}

1. Staicus C, Calina D, Rosu L, Rosu AF and Zlatian O: Involvement of microbial flora in aetiology of surgical site infections. Eur J Hosp Pharm Sci Pract 22: A59, 2015.

2. Watanabe A, Kohnoe S, Shimabukuro R, Yamanaka T, Iso Y, Baba H, Higashi H, Orita H, Emi Y, Takahashi I, et al: Risk factors associated with surgical site infection in upper and lower gastrointestinal surgery. Surg Today 38: 404-412, 2008.

3. Owens CD and Stoessel K: Surgical site infections: Epidemiology, microbiology and prevention. J Hosp Infect 70 (Suppl 2): 3-10, 2008.

4. Cristea OM, Zlatian OM, Dinescu SN, Avramescu CS Balasoiu M, Niculescu M and Calina DC: A comparative study on antibiotic resistance of Klebsiella strains from surgical and intensive care wards. Curr Heal Sci 42: 169-179, 2016.

5. Dulon M, Haamann F, Peters C, Schablon A and Nienhaus A: MRSA prevalence in European healthcare settings: a review. BMC Infect Dis 11: 138, 2011.

6. House of Commons Public Accounts Committee: Reducing Healthcare associated infection in hospitals in England. Fifty-second Report of Session 2008-09. http://www.publications. parliament.uk/pa/cm200809/cmselect/cmpubacc/812/812.pdf. Accessed November 10, 2009.

7. Kassim A, Omuse G, Premji Z and Revathi G: Comparison of Clinical Laboratory Standards Institute and European Committee on Antimicrobial Susceptibility Testing guidelines for the interpretation of antibiotic susceptibility at a University teaching hospital in Nairobi, Kenya: a cross-sectional study. Ann Clin Microbiol Antimicrob 15: 21, 2016.

8. Li JY, Ma Y, Sun Z, Yao L, Zhang L, Hu C and Jin S: Cluster analysis to detect the homogeneous strains of E. coli isolated from clinical specimens. Chin J Microbiol Immunol 23: 384-387, 2003.

9. Xu J, Shi C, Song M, Xu X, Yang P, Paoli G and Shi X: Phenotypic and genotypic antimicrobial resistance traits of foodborne Staphylococcus aureus isolates from Shanghai. J Food Sci 79: M635-M642, 2014.

10. Dzidic S and Bedeković V: Horizontal gene transfer-emerging multidrug resistance in hospital bacteria. Acta Pharmacol Sin 24: 519-526, 2003.

11. Liapakis IE, Kottakis I, Tzatzarakis MN, Tsatsakis AM, Pitiakoudis MS, Ypsilantis P, Light RW, Simopoulos CE and Bouros DE: Penetration of newer quinolones in the empyema fluid. Eur Respir J 24: 466-470, 2004.

12. Kumar M, Dutta R, Saxena S and Singhal S: Risk Factor Analysis in Clinical Isolates of ESBL and MBL (Including NDM-1) Producing Escherichia coli and Klebsiella Species in a Tertiary Care Hospital. J Clin Diagn Res 9: DC08-DC13, 2015. 
13. Neonakis IK, Baritaki S, Georgiladakis A and Spandidos DA Analysis of the beta-lactam resistance phenotypes of Escherichia coli. An 8-year survey conducted in Greece. Roum Arch Microbiol Immunol 67: 10-13, 2008.

14. Neonakis IK, Samonis G, Messaritakis H, Baritaki S, Georgiladakis A, Maraki S and Spandidos DA: Resistance status and evolution trends of Klebsiella pneumoniae isolates in a university hospital in Greece: Ineffectiveness of carbapenems and increasing resistance to colistin. Chemotherapy 56: 448-452, 2010.

15. Sifakis S, Angelakis E, Makrigiannakis A, Orfanoudaki I, Christakis-Hampsas M, Katonis P, Tsatsakis A and Koumantakis E: Chemoprophylactic and bactericidal efficacy of $80 \mathrm{mg}$ gentamicin in a single and once-daily dosing. Arch Gynecol Obstet 272: 201-206, 2005.

16. Petrakis I, Gonianakis C, Vrachassotakis N, Tsatsakis A, Vallilakis IS and Chalkiadakis G: Prospective study of preincisional intraparietal single-dose ceftriaxone in reducing postoperative wound infection in type I and II diabetic patients. Acta Diabetol 36: 159-162, 1999.

17. Neonakis IK, Spandidos DA and Petinaki E: Is minocycline a solution for multidrug-resistant Acinetobacter baumannii? Future Microbiol 9: 299-305, 2014.

18. Youinou P, Garré M, Menez JF, Morin JF and Masse R : Protein malnutrition and deficient cell-mediated immunity in intensive care (author's transl). Nouv Presse Med 10: 3835-3837, 1981 (In French).

19. Neonakis IK, Spandidos DA and Petinaki E: Confronting multidrug-resistant Acinetobacter baumannii: A review. Int J Antimicrob Agents 37: 102-109, 2011

20. Tanase A, Colita A, Ianosi G, Neagoe D, Branisteanu DE, Calina D, Docea AO, Tsatsakis A and Ianosi SL: Rare case of disseminated fusariosis in a young patient with graft vs. host disease following an allogeneic transplant. Exp Ther Med 12: 2078-2082, 2016.

21. Malini A, Deepa E, Gokul B and Prasad S: Nonfermenting gram-negative bacilli infections in a tertiary care hospital in kolar, karnataka. J Lab Physicians 1: 62-66, 2009.

22. Solomkin JS: Antibiotic resistance in postoperative infections. Crit Care Med 29 (Suppl 4): N97-N99, 2001.

23. Cockerill FR, Patel JB: M100-S25 Performance Standards for Antimicrobial Susceptibility Testing; Twenty-Fifth Informational Supplement. Clin Lab Stand Inst 35: 44-49, 2015.
24. Schentag JJ, Hyatt JM, Carr JR, Paladino JA, Birmingham MC, Zimmer GS and Cumbo TJ: Genesis of methicillin-resistant Staphylococcus aureus (MRSA), how treatment of MRSA infections has selected for vancomycin-resistant Enterococcus faecium, and the importance of antibiotic management and infection control. Clin Infect Dis 26: 1204-1214, 1998.

25. Manian FA, Meyer PL, Setzer J and Senkel D: Surgical site infections associated with methicillin-resistant Staphylococcus aureus: Do postoperative factors play a role? Clin Infect Dis 36: 863-868, 2003

26. Taiwo SS: Methicillin resistance in Staphylococcus aureus: A review of the molecular epidemiology, clinical significance and laboratory detection methods. West Afr J Med 28: 281-290, 2009.

27. Ianoşi S, Ianoşi G, Neagoe D, Ionescu O, Zlatian O, Docea AO, Badiu C, Sifaki M, Tsoukalas D, Tsatsakis AM, et al: Age-dependent endocrine disorders involved in the pathogenesis of refractory acne in women. Mol Med Rep 14: 5501-5506, 2016.

28. Thornsberry C and McDougal LK: Successful use of broth microdilution in susceptibility tests for methicillin-resistant (heteroresistant) staphylococci. J Clin Microbiol 18: 1084-1091, 1983.

29. Hiramatsu K, Kihara $\mathrm{H}$ and Yokota T: Analysis of borderline-resistant strains of methicillin-resistant Staphylococcus aureus using polymerase chain reaction. Microbiol Immunol 36: 445-453, 1992.

30. Høiby N, Jarløv JO, Kemp M, Tvede M, Bangsborg JM, Kjerulf A, Pers C and Hansen H: Excretion of ciprofloxacin in sweat and multiresistant Staphylococcus epidermidis. Lancet 349: 167-169, 1997.

31. Peterson LR, Quick JN, Jensen B, Homann S, Johnson S, Tenquist J, Shanholtzer C, Petzel RA, Sinn L and Gerding DN: Emergence of ciprofloxacin resistance in nosocomial methicillin-resistant Staphylococcus aureus isolates. Resistance during ciprofloxacin plus rifampin therapy for methicillin-resistant S aureus colonization. Arch Intern Med 150: 2151-2155, 1990.

32. Tarale P, Gawande S and Jambhulkar V: Antibiotic susceptibility profile of bacilli isolated from the skin of healthy humans. Braz J Microbiol 46: 1111-1118, 2015.

33. Berge AC, Atwill ER and Sischo WM: Assessing antibiotic resistance in fecal Escherichia coli in young calves using cluster analysis techniques. Prev Vet Med 61: 91-102, 2003. 